and the demonstration that single somite defects could impair septation of the outlet of the heart and produce perimembranous ventricular septal defects, fit together beautifully the clinical defects and the possible sites of gene action. She too used quail chimeras to demonstrate neural crest migration into the cardiac outlet, this time with chicken embryos rather than Mark Ferguson's aligators (quickens not qualigators). My one criticism was of the suggestion in one report that because absence of the spleen occurred with outlet defects of the heart, the neural crest might contribute to spleen formation. This is the old error of confusing association with cause. There is impressive evidence to show that absence of the spleen in the Ivemark syndrome results from a disturbance of laterality such that the body is isomeric with two right sides. My own preference is, therefore, for the term isomerism sequence but this debate highlights the danger of such terminology based on an interpretation of embryology. Are all cleft palates in babies with small jaws truly sequential as the Robin sequence would have us believe? We should, perhaps, heed cardiac anatomists and embryologists who have purged their terminology of such assumptions and restrict themselves to actual observations. Long live the straight forward eponymous syndrome.

I hope that most of the participants returned home with happy memories, particularly of the 'special item'. Together with Carol McKeown and Di Donnai I ended the unknown syndrome session with a test of the dysmorphologists' skills. They were shown pictures of themselves at a very early age to see if they could identify which furrowed face had evolved from which picture of innocence. It proved very entertaining since few of the victims realised that their spouses had betrayed them. Needless to say all copies of these slides have now been incinerated.

JOHN BURN

Department of Human Genetics, University of Newcastle upon Tyne, 19 Claremont Place, Newcastle upon Tyne $N E 24 A A$.

\title{
Second International Workshop on Multiple Endocrine Neoplasia Type 2 (MEN 2), Cambridge, 17 to 20 September 1986
}

MEN 2 is an autosomal dominant inherited cancer syndrome. It consists of medullary thyroid carcinoma (MTC), which arises from the calcitonin producing thyroid $\mathrm{C}$ cells, and phaeochromocytoma of the adrenal medulla. The five sessions of the Workshop reflected the diversity of scientific and clinical interest in this syndrome.

The first session dealt with radioimmunoassays and family screening. Both here and in a concluding round table, the consensus was that screening should be offered not only to members of known families, but to first degree relatives of patients with apparently sporadic MTC. The incomplete penetrance of the MEN 2 gene means that a negative family history may be misleading (Ponder, Sutton). Calcitonin remains the only satisfactory marker for screening for MTC; katacalcin values run parallel but offer no advantage, CGRP (calcitonin gene related peptide) seems not to be useful, and chromogranin A has yet to be fully evaluated. A review of European calcitonin assays (Gautvik,
Oslo) showed alarming variation in the results obtained with a standard panel of samples: most groups with experience in screening cross check their results in a second assay before advising thyroidectomy.

Genetic linkage studies have not so far produced a positive result. About $30 \%$ of the genome has been excluded (results collected by K Kidd (Yale) and N Simpson (Kingston)). More data was presented by the Detroit group in favour of the $20 \mathrm{p}$ - deletion, but this has not been seen by other laboratories, and a polymorphic DNA probe mapped by in situ hybridisation close to the supposed deleted band gives a strongly negative lod score. Flanking probes for the deletion have not, however, been identified.

Cell cultures derived from MTC have provided the opportunity to study the regulation of calcitonin (CT) gene expression, and of the differential splicing of the CT/CGRP precursor mRNA. Modulation of CT gene expression during growth of MTC cells in culture may reflect changes in the production of 
$\mathrm{CT}$ and other peptides during evolution of tumours in vivo (Baylin, Baltimore).

An unresolved problem in the pathology of MTC is the coexistence in some tumours of both calcitonin producing cells and typical thyroid follicular epithelial cells. While $C$ cells are thought to be of neuroectodermal origin, differentiation of a subset of $\mathrm{C}$ cells from thyroid epithelium (endodermal origin) remains a possibility. Several papers were presented showing the results of in situ hybridisation using cDNA and cRNA probes, in some cases combined with immunohistochemistry for localisation of gene products (Williams, Cardiff; Wolfe, Boston), applied to a variety of endocrine peptides and oncogenes. There was considerable optimism that these techniques would allow a more precise description of the phenotype and lineage of normal and neoplastic $\mathrm{C}$ cells than has so far been available.

The problem of hyperplasia of C cells and of phaeochromocytomas was also discussed. There is strong evidence that hyperplasia of both cell types precedes neoplasia in MEN 2, but the clear separation of genetically determined hyperplasia from the occasional focal hyperplasia in apparently unaffected persons remains a difficulty.

The clinical sessions included data on the lack of ill effects of thyroidectomy in childhood (Telander, Mayo Clinic), a new exercise test with measurement of urinary epinephrine:dopamine ratio to discriminate patients with early phaeochromocytomas from normal controls and patients who are simply anxious (Telenius-Berg, Hassleholm), and data on imaging techniques in the diagnosis of MTC, of which the new screening agent pentavalent DMSA may be the most promising (Clarke, London). Finally, Gageథ (Houston) presented the Boston experience of 1 . years follow up of screening in the ' $\mathrm{J}$ ' kindred $\overline{\text { s }}$. originally reported in 1971 . The data provide evidence (1) that screening leads to early diagnosise and that this is curative; (2) in all but two cases? thyroid $\mathrm{C}$ cell abnormalities were detected b 9 screening before signs of adrenal medullary disease $\overrightarrow{\dot{\vec{H}}}$ and (3) that unilateral phaeochromocytoma caw safely be managed by unilateral adrenalectomy. $\overline{\bar{z}}$

The Workshop, organised by the Cancer Re? search Campaign Medullary Thyroid Group attracted 80 participants, the majority from outside the UK. A convivial atmosphere, helped by brillian September sunshine, stimulated lively discussions at the end of every session and long into the evenings? The next decisive event in the clinical and scientific study of MEN 2 will be the finding of linkage, an then the gene itself. In confident anticipation, the Third Workshop is planned for 1989.

B Ponder* AND E D Williams *The Institute of Cancer Research Royal Cancer Hospitalo The Haddow Laboratories Sutton, Surrey SM2 5PX and the Department of Pathology University of Wales College of Medicine Heath Park $\overrightarrow{\overrightarrow{0}}$ Cardiff CF4 $4 X N$ 